\section{WELTWASSERKONGRESS 2008}

\section{Auszeichnung für Projekte der LINZ AG}

Im Rahmen des Weltwasserkongresses im September 2008 in Wien wurden zwei Projekte der LINZ AG ausgezeichnet: Das „Infocenter Wasserwelt Scharlinz" entschied die Kategorie Customer Services Activities des „IWA Marketing und Communications Award“ für sich. Das idyllisch im Linzer Wasserschutzgebiet „Wasserwald" gelegene denkmalgeschützte Wasserwerk Scharlinz bietet in der Ausstellung rund um das Thema Wasser Interessenten aller Altersgruppen Einblicke in die Welt des Wassers. In der Wasserwelt Scharlinz können Besucher den Weg des Trinkwassers vom Ursprung bis zum Wasserhahn und wieder zurück zur Natur verfolgen. Mit dem IWA Marketing and Communications Award werden Projekte ausgezeichnet, die eine nachhaltige Meinung über Wasser als essenzielles Gut fördern. Weiters ist es das Ziel des Preises, das weltweite Bewusstsein für die wachsende Bedeutung einer professionellen und effektiven Kommunikation zwischen Wasserversorgern und -verbrauchern zu stärken. Der Bereich Abwasser gewann mit dem Projekt für innovative Abwasserentsorgung in der solarCity den „Project Innovation Award" der IWA auf europäischer Ebene. Der von der IWA alle zwei Jahre vergebene PIA zeichnet weltweit hervorragende und innovative Wasserprojekte aus. Die Bewertung der eingereichten Projekte wird nach fünf Kriterien vorgenommen. An erster Stelle steht dabei die erstmalige und innovative Anwendung neuer oder bereits existierender Technologien. Neben dem zukünftigen Wert für die Wasserbranche sowie dem sozialen, ökologischen und nachhaltigen Design wird auch die Komplexität des vorliegenden Problems bewertet, zu dessen Lösung das Projekt beitragen soll. Das Projekt der alternativen Abwasserentsorgung in der Linzer solarCity mit dem Ziel, die Nährstoffkreisläufe zu schließen, indem in 88 Wohnungen, der Volksschule und dem Kindergarten insgesamt 127 Separationstoiletten zur Trennung von Flüssig- und Feststoffen beim Spülgang eingebaut wurden, überzeugte die Jury.

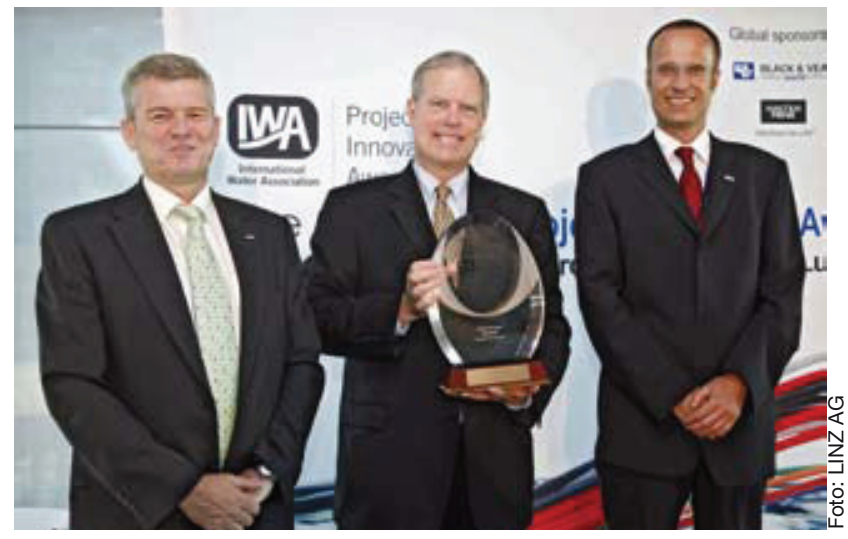

(v.l.n.r.): GF DI Alfred Leimer (LINZ AG), Bill Dee (Chair of the American Academy of Environmental Engineers) und DI Dr. Peter Schweighofer (LINZ AG) 


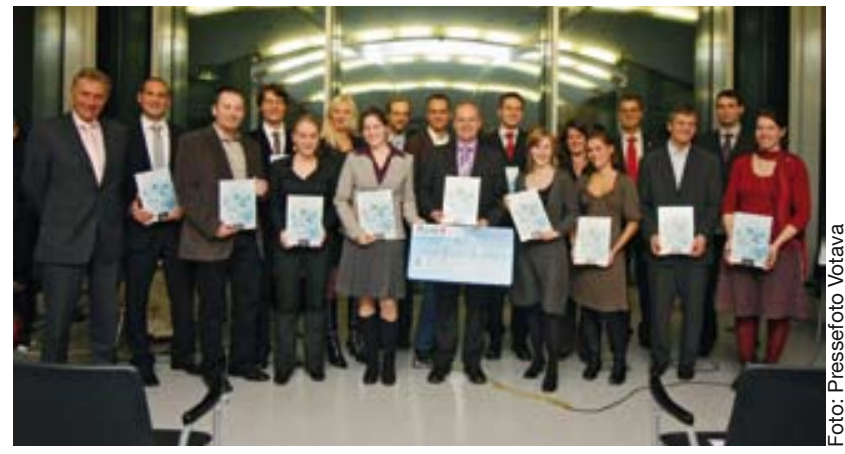

(v.l.n.r.): DI Hans Sailer, Leiter der Wiener Wasserwerke (MA 31) und Ing. Wolfgang Khutter, stv. Leiter der MA 22 mit den Preisträgerlnnen 2008

\section{ENTSORGUNGSBETRIEBE SIMMERING}

\section{Christian Gantner neuer Direktor der EbS GmbH}

(RK) Die Entsorgungsbetriebe Simmering GmbH (EbS), eine 100-prozentige Tochter der Stadt Wien, deren Hauptaufgabe in der Betriebsführung der Hauptkläranlage Wien liegt, steht ab kommendem Jahr unter neuer Leitung: Nach öffentlicher Ausschreibung der Position und einstimmiger Empfehlung der externen Hearing-Kommission wird Ing. Christian Gantner neuer Direktor der EbS. Der 1959 in Wien geborene Christian Gantner absolvierte die HTL Wien III Leberstraße, Fachrichtung Bautechnik. Nach mehreren Jahren in der Privatwirtschaft, wo er in den Bereichen Abwassertechnik und Straßenbau tätig war, begann Gantner 1987 seine Karriere bei der Stadt Wien, zunächst in der MA 30 - Wien Kanal. 1994 wechselte Christian Gantner in die Stadtbaudirektion, Gruppe Umwelttechnik und technische Betriebe. Dort war er unter anderem in die Planungsphase zum Ausbau der Erweiterung der Hauptkläranlage und in die
Budgeterstellung für die umwelttechnischen Dienststellen der Stadt Wien eingebunden. Mit 1. Mai 2001 wurde Gantner in die Geschäftsgruppe Umwelt berufen, wo er die Budgetkoordination verantwortete und damit auch für die Finanzierung der EbS GmbH zuständig war. Anfang 2002 wurde Christian Gantner zusätzlich zum Büroleiter der Geschäftsgruppe Umwelt ernannt. In der EbS GmbH war er unter anderem ab 2005 als Mitglied des Aufsichtsrates tätig. Die 1976 gegründete Entsorgungsbetriebe Simmering GmbH steht zu 100 Prozent im Eigentum der Stadt Wien. In deren Auftrag führt sie die Hauptkläranlage Wien in Simmering, in der das Abwasser aller Wienerinnen und Wiener geklärt wird.

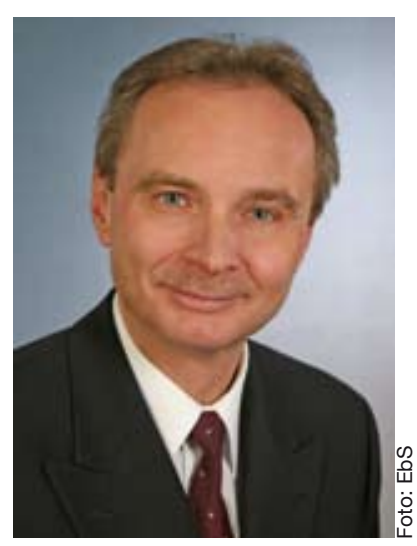

Ing. Christian Gantner, Direktor der EbS ab 2009

\section{UMWELTSCHUTZ}

\section{Wissenschaftlicher Förderpreis 2008 der MA 22 vergeben}

\section{(RK) Am 29. Oktober 2008} wurde der wissenschaftliche Förderpreis für neue Ideen zum Umweltschutz von der MA 22 - Wiener Umweltschutzabteilung im Media Tower Wien vergeben. Die Wiener Wasserwerke (MA 31) beteiligten sich heuer zum zweiten Mal an der Initiative der Wiener Umweltschutzabteilung mit der Ausschreibung eines „Sonderpreises Wasser“. Bei den eingereichten Arbeiten handelt es sich um Diplomarbeiten oder Dissertationen, die thematisch für den Umweltschutz in Wien von Interesse sind. Dr. Karin Büchl-Krammerstätter, Leiterin der Wiener Umweltschutzabteilung, DI Hans Sailer, Leiter der Wiener Wasserwerke und Dr. Ingela Bruner, Rektorin der Universität für Bodenkultur Wien, überreichten die Urkunden und Preisgelder in der Höhe von je 625 Euro. „Fundierte wissenschaftliche Erkenntnisse sind die Basis für strategisches Handeln im Umweltschutz. Die Wiener Umweltschutzabteilung arbeitet in allen Bereichen des Umweltschutzes, sei es Naturschutz, Luftgüte oder Abfall- und Ressourcenmanagement, mit WissenschafterInnen zusammen. Mit dem Förderpreis wollen wir gerade der junge Generation von WissenschafterInnen den Zugang zur praktischen Umweltschutzarbeit erleichtern und sie in ihrem Engagement für den Umweltschutz bestätigen“, so Dr. Karin BüchlKrammerstätter. „Die Stadt Wien unternimmt alles, um das lebensnotwendige Gut Wasser keiner Gefährdung auszusetzen und um die hohe Qualität des Wiener Wassers auch für zukünftige Generationen zu erhalten. Die Wissenschaft ist uns bei unseren Maßnahmen dafür eine unverzichtbare Partnerin", so der Leiter der Wiener Wasserwerke DI Hans Sailer.

\section{KOMMUNALE ABFALLWIRTSCHAFT}

\section{Jahre OÖ. Landesabfallverband}

Am 15. Oktober 2008 feierte der OÖ. Landesabfallverband in Linz sein 15-jähriges Bestehen. Im Jahre 1993 als freiwilliger Zusammenschluss der 15 Bezirksabfallverbände und Statutarstädte gegründet, hat sich der OÖ. LAV in den letzten 15 Jahren zu einer zentralen Drehscheibe der kommunalen Abfallwirtschaft in Oberösterreich entwickelt. Diese 15 Jahre waren die Zeit einer völligen Wende in der Abfallwirtschaftspolitik Oberösterreichs - vom Müllnotstand und der Verwertung nur eines Drittels des anfallenden Abfalls hin zu vorbildlichen $68 \%$, die heute verwertet werden. Damit konnten Kosten wie Umweltbelastung massiv verringert werden. In 185 Altstoffsammelzentren werden mittlerweile mehr Altstoffe gesammelt (184.000 t) als Restabfälle im ganzen Bundesland anfallen (169.000 t). Der Landesabfallverband ist ein wichtiger Faktor in dieser Erfolgsgeschichte - er garantiert eine konsequente Umsetzung der Abfallwirtschaftspolitik, Koordination und Kontinuität über alle Bezirke. Bgm. Josef Moser, Vorsitzender des OÖ. Landesabfallver- 


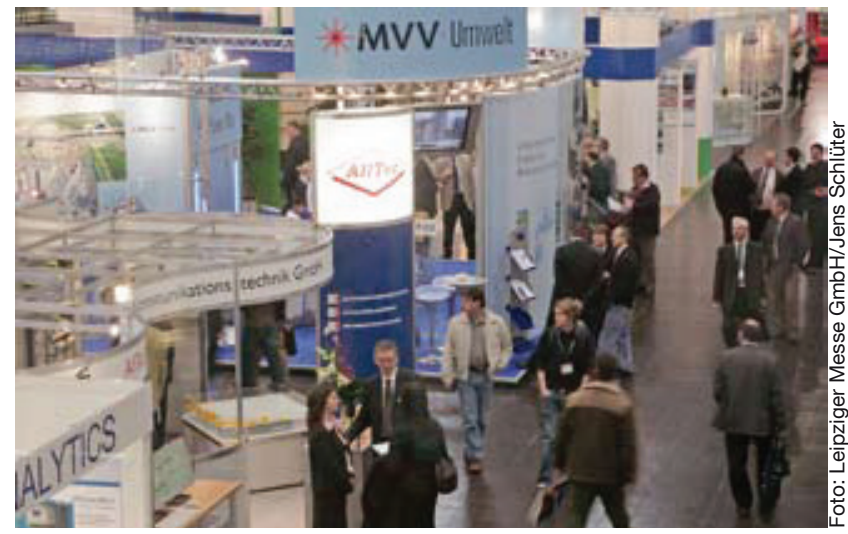

bandes: „Der OÖ. Landesabfallverband gewährleistet den Informationsaustausch, zahlreiche Synergien in der gemeinsamen Projektabwicklung und koordiniert auch die strategische Weiterentwicklung der öffentlichen Abfallwirtschaft. Der LAV sucht für jede Aufgabe die ökologisch und ökonomisch effizienteste Art der Umsetzung."

\section{FACHMESSE}

\section{TerraTec schlägt} Brücke zum Wachstumsmarkt Osteuropa

Mit 22 Millionen Einwohnern ist Rumänien der zweitgrößte osteuropäische EU-Mitgliedsstaat. Das Land verzeichnete in den vergangenen Jahren ein rasantes Wirtschaftswachstum. Um den steigenden Energieund Ressourcenbedarf, einen höheren Lebensstandard und wachsende Produktionsraten nachhaltig und umweltverträglich auszugestalten, steht die rumänische Politik vor der großen Aufgabe, eine entsprechend anspruchsvolle Umweltgesetzgebung vor dem Hintergrund des geltenden EURechts zu erarbeiten. Bis 2017 müssen 251 Deponien, die nicht den EU-Anforderungen genügen, geschlossen und gleichzeitig neue Entsorgungsmöglichkeiten geschaffen werden. In der Wasserwirtschaft stehen der Bau neuer Abwasserbehandlungsanlagen und die Modernisierung bestehender Anlagen an. Bisher sind nur 52 Prozent der Kommunen an das Wasser- und Abwassernetz angeschlossen. 2007 wurde mit der Erneuerung der Wasser- und Abwassersysteme in 1.200 Dörfern begonnen. Für 60 Großprojekte wurde bei der EU finanzielle Förderung beantragt. Dafür besteht auf Auftraggeber- wie auf Auftragnehmerseite viel Informations- und Gesprächsbedarf, dem die TerraTec, die von 27. bis 29. Januar 2009 in Leipzig veranstaltet wird, mit ihrem speziellen Fachprogramm ein optimales Forum bietet. Um die unterschiedlichen Möglichkeiten der Unterstützung von Umweltprojekten in Osteuropa geht es beim internationalen Kontaktforum der TerraTec. Außerdem werden Investitionsbedarf, Markteintrittsmöglichkeiten und Rahmenbedingungen in den Bereichen Abfall-, Wasser- und Energiewirtschaft unter die Lupe genommen.

\section{Weitere Informationen:} www.terratec-leipzig.de 